\title{
Compte rendu du colloque “L’enseignement des statistiques en sciences humaines et sociales”
}

\author{
Catherine Lemay ${ }^{\mathrm{a},} \otimes$ and William Laporte ${ }^{\mathrm{b}}$ \\ ${ }^{a}$ Université du Québec à Chicoutimi \\ ${ }^{\mathrm{b}}$ Université du Québec à Trois-Rivières
}

\begin{abstract}
Ce texte présente un compte rendu du colloque sur l'enseignement des statistiques en sciences humaines et sociales qui s'est tenu à Chicoutimi le 10 mai 2018 dans le cadre de la rencontre annuelle de l'Association francophone pour le savoir (ACFAS). Cinq conférenciers ont discuté des aspects liés à l'enseignement des statistiques. Yves Lacouture a fait un état de la situation dans plusieurs universités québécoises; Eugénie Dostie-Goulet a présenté les quatre clés pour réussir à enseigner les statistiques, soit la finalité, l'approche, le contenu et le processus; Sébastien Béland a discuté de la mesure de la statisticie; Daniel Lalande a énuméré différents facteurs psychologiques influençant l'apprentissage des statistiques, de l'anxiété à la motivation en passant par les buts et les attitudes; Denis Cousineau a argumenté que la source de l'anxiété statistique et la difficulté à apprendre les statistiques en général proviennent d'aptitudes cognitives réduites pour le visuospatial; finalement, William Laporte a présenté les résultats d'une étude sur des enseignants de statistique pour mettre à jour les concepts essentiels. // This text is a proceeding of the symposium on the teaching of statistics for social sciences and humanities held in Chicoutimi on May 10th, 2018 as part of the annual meeting of the Association francophone pour le savoir (ACFAS). Five presenters discussed of topics related to teaching of statistics. Yves Lacouture presented an overview of the situation in many Québec universities; Eugénie Dostie-Goulet introduced the four keys for successful teaching, finality, approach, content, process. Sébastien Béland explored measures of statisticy; Daniel Lalande enumerated psychological factors affecting learning of statistics, from anxiety to motivation, including goals and aptitudes; Denis Cousineau argued that the origin of statistics anxiety and statistics learning is to be found in reduced visuo-spatial abilities; finally, William Laporte presented results on teachers of statistics in order to uncover the essential concepts of statistics.
\end{abstract}

Keywords $\backsim$ Statistics teaching; proceedings; ACFAS 2018.

Acting Editor $=$ Denis Cousineau (Université d'Ottawa)

\section{Introduction}

Ce texte est un compte rendu de la rencontre tenue le 10 mai 2018 à Chicoutimi dans le cadre de l'ACFAS. Il a adopté un format chronologique, présentant les conférences dans l'ordre où elles ont eu lieu. À la fin de chaque section, un sommaire des questions posées est donné.

\section{Réflexions sur l'enseignement des statistiques}

Cette présentation d'Yves Lacouture (Université Laval) avait pour but de donner l'état de la situation actuelle concernant l'enseignement universitaire des statistiques dans les programmes de sciences humaines et sociales. Le département de psychologie de l'Université Laval avait créé, en 2017, un comité de réflexion sur l'enseignement des statistiques. Le rapport a été présenté en juin la même 
année. Pour ce faire, Y. Lacouture et ses collègues ont consulté les plans des cours de statistique ainsi que les descriptions officielles, et ce pour plusieurs universités afin d'être informés des concepts enseignés dans les programmes de psychologie (1er, 2e et 3e cycle). Également, ils ont suggéré un degré de littéracie en statistique que les étudiants devraient acquérir. De plus, il y a eu des réflexions portant sur les différentes méthodes d'enseignement soit en présentiel ou à distance. Finalement, ils ont voulu déterminer la place des logiciels dans l'enseignement des statistiques à l'école de psychologie de l'université Laval.

Les universités qui constituent l'échantillon des programmes de psychologie sont les universités Laval, McGill, Montréal, Ottawa, Sherbrooke, UQAM et UQTR. L'inventaire des contenus enseignés (voir la figure 1 à la page suivante) est composé de quatre blocs soient les fondements (l'ensemble des connaissances préalables à la compréhension des statistiques), les statistiques de base (univariées et bivariées), l'analyse de plans d'expériences (une variable dépendante) et les analyses multivariées (plusieurs variables dépendantes). Le premier bloc est traité dans le cours de CÉGEP préalable à l'admission en psychologie. Quant au deuxième bloc, celui-ci est traité dans le cours de statistique obligatoire au premier cycle en psychologie. Le troisième bloc est généralement enseigné dans un cours optionnel en statistique au 1er cycle en psychologie. Le quatrième bloc est abordé aux cycles supérieurs en psychologie. Les étudiants qui aspirent à la profession de psychologue devraient avoir une connaissance de base des blocs 1 à 3 et un aperçu du bloc 4 . Également, les étudiants qui désirent poursuivre leur formation universitaire en recherche devraient minimalement maîtriser les quatre blocs de contenu. Ces connaissances sont nécessaires afin d'être un lecteur averti et facilitent la compréhension des données probantes ainsi que la poursuite de la formation continue. Par contre, il existe un paradoxe qui se traduit par une hausse des qualifications requises et un manque d'accessibilité de la formation en statistique. Actuellement, il est dit qu'il y a un manque de connaissances en statistique pour les chercheurs en formation qui se retrouvent souvent à être formés "sur le tas".

Quelques recommandations ont été effectuées pour l'école de psychologie de l'université Laval. Selon Y. Lacouture, il faut maintenir le mode présentiel pour l'ensemble des cours de statistiques offerts. De plus, il serait pertinent de revoir le cours optionnel au 1er cycle afin d'offrir un plus grand ensemble de contenus statistiques. Également, il faudrait encourager les étudiants de 1er cycle à suivre le cours optionnel en statistique qui était autrefois obligatoire pour l'admission au doctorat. L'adoption du logiciel SPSS comme logiciel standard et son utilisation dans tous les cours de statistiques serait une autre recommandation intéressante. Le maintien des cours de plans d'expériences (bloc 3) et multivariés (bloc 4) aux cycles supérieurs est important et il serait pertinent de revoir la structure des programmes pour qu'ils puissent être suivis. La proposition d'un séminaire de $2 \mathrm{e}$ et $3 \mathrm{e}$ cycle en statistiques de pointe portant sur des contenus variables et pouvant être crédité dans la formation a été soulevée par le comité.

\section{La période de questions}

1. Quand est-il des écoles d'été en statistique?

Il y a déjà eu une tentative d'implantation d'une école d'été en statistique à l'université Laval, mais ce projet n'a pu perdurer dans le temps pour des raisons financières.

2. Pourquoi pas un mode d'enseignement hybride comme recommandation pour les cours de statistique plutôt que présentiel?

Les cours de type présidentiel contribuent à favoriser la motivation des étudiants qui est moindre au début du cours de statistique. La présence de l'enseignant permet d'abaisser l'insécurité associée aux statistiques et le dynamisme de ce dernier peut influencer l'expérience académique de l'étudiant. Par contre, il serait pertinent de discuter d'un type de cours qui comprendrait des cours présidentiels et une partie du cours où l'étudiant serait amené à travailler de la maison afin de développer son autonomie par le biais du cours.

3. Les élèves du primaire débutent leurs apprentissages des fondements en statistique de plus en plus tôt dans leurs parcours académiques. Quel impact cela aura sur l'éducation future? Va-t-on les accueillir de la même façon lors de leur entrée universitaire dans les cours de statistique?

Il est dit que plus les étudiants acquièrent tôt les connaissances relatives aux fondements des statistiques, plus ils sont familiers avec les concepts de base de cette discipline. Il n'en demeure pas moins qu'il est important de revenir sur ces derniers lors du premier cours obligatoire de statistique en psychologie. Par contre, le poids associé à ces concepts est discutable et reste à valider lors de l'élaboration du plan de cours.

\section{Enseigner les statistiques : les clés de la réussite}

Cette présentation d'Eugénie Dostie-Goulet (Université de Sherbrooke) a traité de la réalité actuelle du cours de statistique devenu obligatoire à la maîtrise de l'école de politique appliquée de l'Université de Sherbrooke depuis 2008, ce qui constitue une rareté dans ce domaine d'étude.

Selon E. Dostie-Goulet, il y a quatre clés de la réussite lorsqu'on enseigne les statistiques, soit la finalité, l'approche, le contenu et le processus. La première clé se traduit par un intérêt au produit des statistiques donc 
FIGURE 1 — Inventaire des concepts statistiques en psychologie

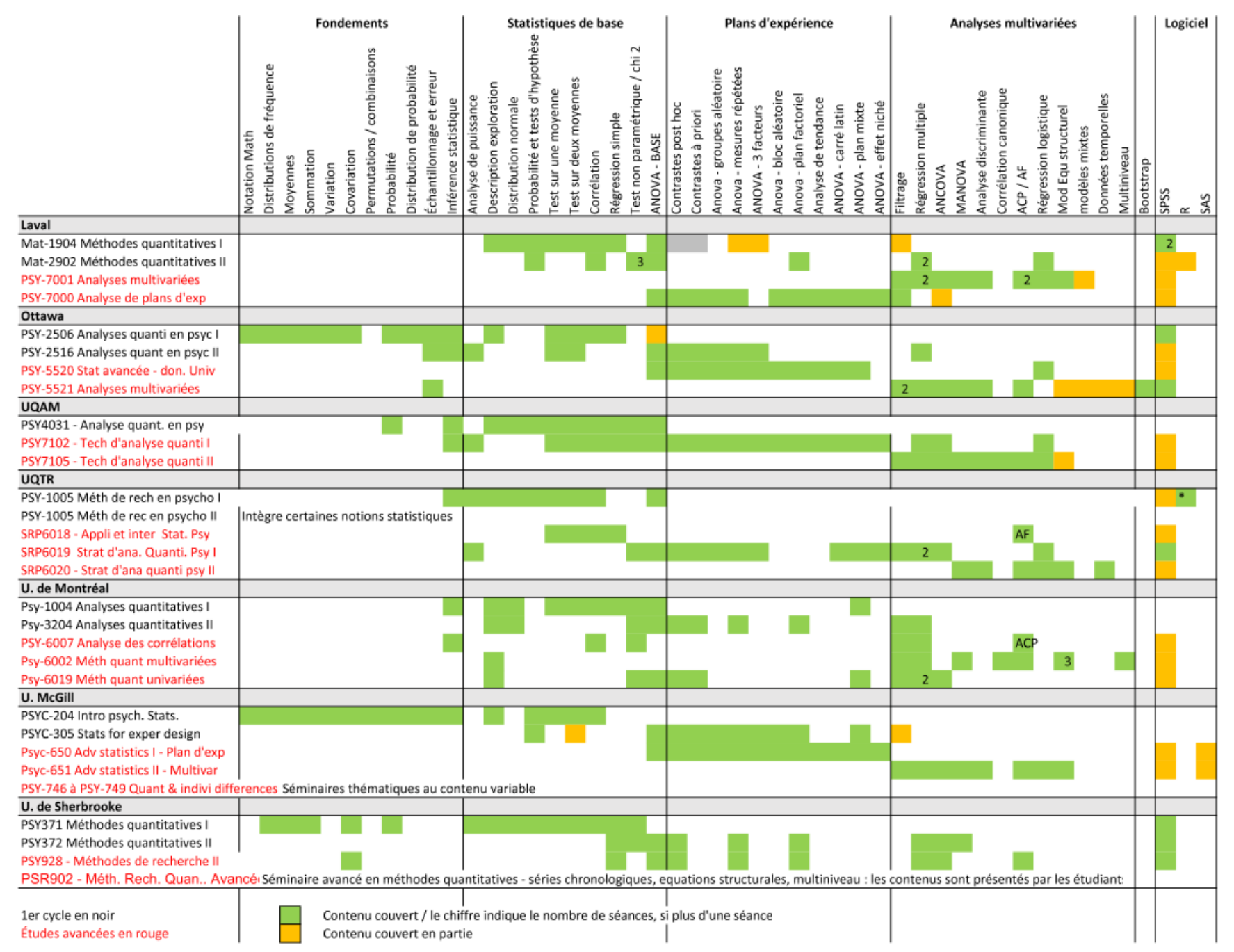

aux conditions d'utilisation, aux objets appropriés, à l'interprétation des résultats ainsi qu'au traitement sous forme de tableaux et de graphiques. On cherche à former des citoyens critiques. Les statistiques ne sont pas un but, mais un outil au service de la compréhension disciplinaire. Dans cette optique, l'outil mathématique est réduit au minimum.

L'approche se définit par des cours plus ou moins magistraux où les discussions sont fréquentes avec des petits groupes d'étudiants. Un total de trois outils sont utilisés dans le cadre du cours pour faciliter la coopération et pour donner la possibilité à l'étudiant de réaliser les exercices et travaux lorsqu'il le souhaite : le site web Moodle, le logiciel Run manuel dédié à la syntaxe de $\mathrm{R}$ et finalement, le site web Dimension. Également, il y a des périodes de laboratoire où les étudiants sont accompagnés dans leurs démarches par l'enseignant et un auxiliaire de cours. Il y a sept travaux pratiques conçus de façon évolutive à effectuer en ligne dans la session (aucune correction pour l'enseignant). Ceux-ci permettent d'évaluer de façon automatique le produit et les connaissances. Par contre, il y a un examen à la fin de la session. De plus, il y a une présentation orale notée qui permet d'échanger sur divers aspects des bases de données et de la présentation de résultats.

Le contenu de ce cours est tiré de données réelles et de difficultés "authentiques" reliées au domaine de la politique qui amènent graduellement les étudiants vers le tra- 
vail pratique. De plus, les exercices et les laboratoires sont créés à partir de six bases de données "réelles". Lors de l'élaboration des travaux pratiques, chaque étudiant doit utiliser deux bases de données "réelles". En ajoutant les présentations orales des étudiants et des enseignants, le groupe est exposé, directement ou indirectement, à une vingtaine de bases de données.

Finalement, le processus (schématisé à la Figure 2) est évalué alors que l'étudiant doit franchir les étapes de l'élaboration d'une recherche qui engendre des défis statistiques, techniques et disciplinaires. Il est important de suivre un processus intégré et interactif pour les étudiants afin de favoriser l'apprentissage. Les défis sont surmontables et amènent les étudiants à se démener et parfois à dépasser le cadre de réflexion. L'évaluation du processus est importante dans le cheminement académique de l'étudiant et permet de le rendre autonome avec les concepts et les démarches statistiques.

\section{La période de questions}

1. Est-ce que le dynamisme de l'enseignant joue un rôle dans la motivation des étudiants quant à la poursuite de ce cours?

C'est l'addition de la personnalité dynamique de l'enseignant et du matériel pédagogique utilisé qui contribue à accroître la motivation de l'étudiant, selon E. DostieGoulet.

2. Comment pourrait-on généraliser cette façon d'enseigner les statistiques à de grands groupes?

La majeure partie de ces clés de réussite pourrait assurément être appliquée à de grands groupes. L'absence de correction par l'élaboration de travaux pratiques en ligne pourrait être adaptée à cette réalité avec des suivis de l'enseignant. La présence d'un assistant de cours lors des laboratoires ainsi qu'un démonstrateur permettant de présenter la démarche appropriée sur le logiciel statistique $R$ reste essentielle. Il n'en demeure pas moins que les conditions gagnantes à la transmission de la matière se traduisent mieux dans de petits groupes d'étudiants avec un suivi de leur cheminement personnalisé.

\section{La mesure de la statisticie : quelques exemples}

Cette présentation de Sébastien Béland (Université de Montréal) a abordé trois aspects de l'enseignement en statistique soit la mesure, la mesure de l'habileté et l'habileté en statistique. En ce qui a trait à la mesure en soi, son résultat est une valeur numérique qui exprime un ratio entre une grandeur étudiée et une grandeur standardisée considérée comme une unité. Concernant la mesure de l'habileté en statistique, il est impossible de l'observer directement chez un individu. On doit l'inférer à partir de variables manifestes tels les items à un test. Actuellement, il y a l'hypothèse que la statistique est conçue comme un construit composé d'items formatifs. C'est du moins ce qui est observé dans certains tests tels celui de Allen, le Assessment resource tools for improving statistical thinking (ARTIST) ou le Statistical Reasoning in Biology Concept Inventory (SRBCI).

Quelques pistes de réflexion ont été abordées afin d'alimenter la deuxième partie de ce colloque. En effet, les tests abordés durant cette présentation sont constitués d'items formatifs. Pourrait-on envisager l'utilisation d'items réflectifs ou postuler l'existence d'une théorie permettant de mesurer l'habileté en statistique? De plus, les publications scientifiques abordent l'habileté en statistique telle un construit multidimensionnel. Pourrait-on croire en l'existence d'une habileté générale en statistique? Généralement, les enseignants en statistique évaluent les étudiants à l'aide d'items formatifs à choix multiples permettant l'évaluation des connaissances de ces derniers. Par contre, il serait pertinent de s'intéresser aux compétences que doivent maîtriser les étudiants en statistique. Une investigation pourrait débuter sur ces compétences de sorte à trouver de nouveaux outils d'évaluation plus adaptés et les tâches proposées aux étudiants deviendraient plus authentiques telles que le test SRBCI. À la lumière de ces interrogations, une liste de compétence essentielle qui servirait de tableau de spécification à des tests pourrait être envisagée afin de mesurer l'habileté (et/ou les compétences) en statistique.

\section{La période de questions}

1. Est-ce que l'habileté statistique est la même pour tous les domaines d'études?

2. Comment distingue-t-on l'habileté statistique de la connaissance?

3. Qu'est-ce que l'on doit enseigner comme matière en statistique à la base?

4. La compréhension du langage est importante à considérer comme habileté en statistique. À titre d'exemple, la notion de hasard est difficilement comprise par les étudiants.

\section{L'apprentissage des statistiques : les facteurs psycholo- giques}

Cette présentation de Daniel Lalande (Université du Québec à Chicoutimi) avait pour but d'effectuer un survol des conséquences et des déterminants de l'anxiété statistique, d'exposer des pistes de recherches futures ainsi que d'aborder des interventions pour améliorer l'apprentissage des statistiques. Le cours de statistique est obligatoire dans plusieurs programmes de sciences humaines et sociales et ce dernier provoque souvent de l'anxiété chez les étudiants. L'anxiété statistique qui est présente chez 66 


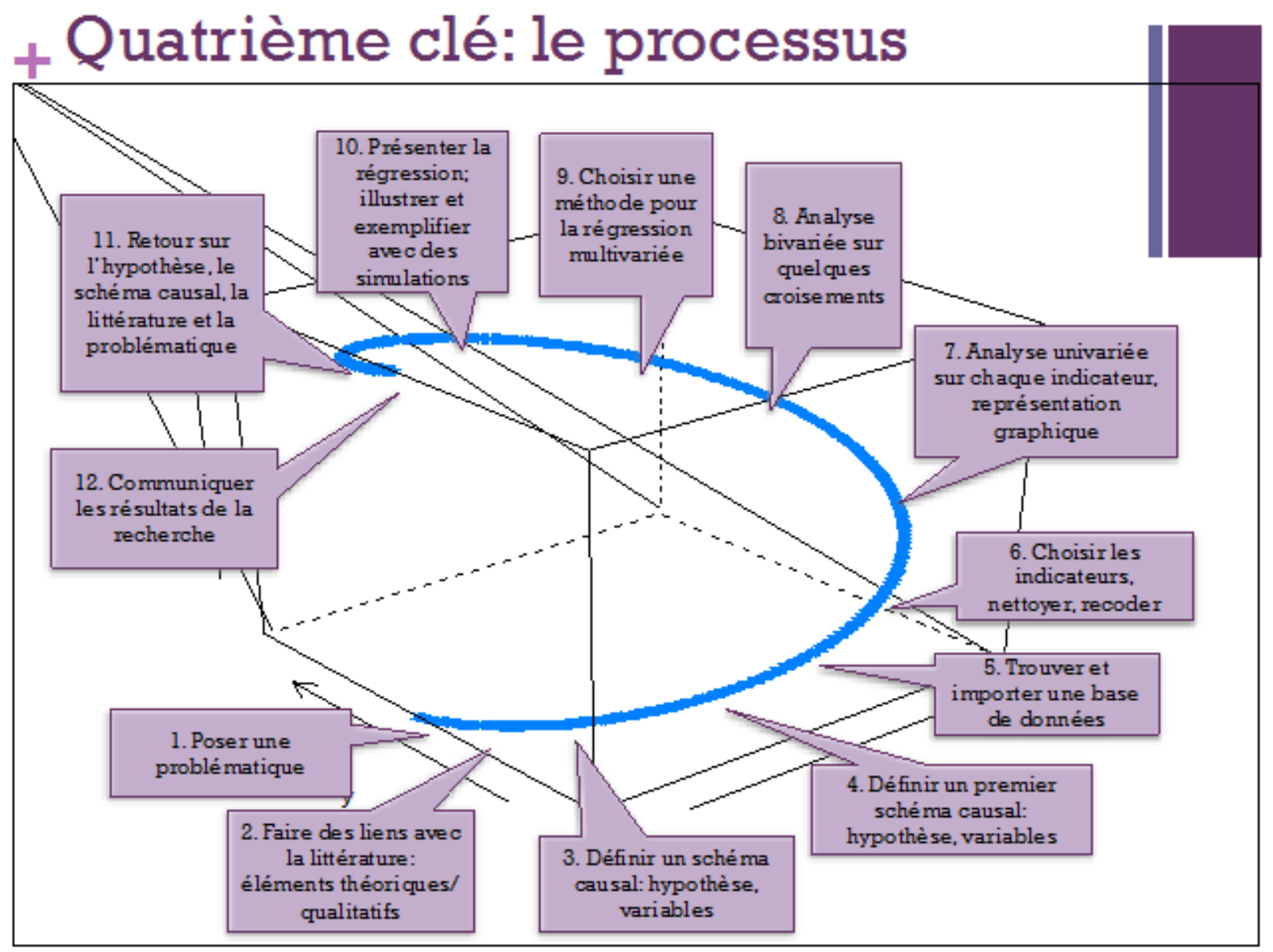

$\%$ à $80 \%$ des étudiants est un construit multidimensionnel qui comprend les dimensions cognitive et affective. L'augmentation de l'anxiété statistique augmente la procrastination et diminue la performance académique.

Il y a plusieurs facteurs psychologiques impliqués dans l'anxiété statistique. Une augmentation de la perception d'auto-efficacité diminuerait l'anxiété. Les attitudes positives à l'égard des statistiques diminueraient également l'anxiété statistique des étudiants. Le fait d'avoir des buts de maîtrise diminuerait l'anxiété statistique alors que les buts de performance (approche ou évitement) augmentent l'anxiété statistique. La Théorie de l'autodétermination (TAD) pourrait permettre de comprendre le type de motivation d'un étudiant face au cours de statistique et cela pourrait constituer des pistes de recherche futures. Plus on tend vers une motivation intrinsèque, plus le comportement de l'étudiant dans le cours de statistique est autodéterminé.
Quelques interventions ont été nommées afin d'améliorer l'apprentissage des statistiques pour changer le rapport des étudiants avec l'anxiété. Il est dit que c'est la composante cognitive qui nuit à la performance académique. Une technique nommée "One minute paper strategy" consiste à inscrire de façon anonyme le concept le plus important que l'étudiant a appris en classe à la fin de chaque cours ainsi que les questions qui demeurent sans réponse. À la fin de la session, les étudiants présentent moins d'anxiété et connaissent une meilleure performance académique.

Quelques recommandations peuvent être mises de l'avant telles l'utilisation de petits groupes pour l'enseignement, changer l'approche des enseignants afind de rendre les cours plus plaisants et accessibles par l'intégration de l'humour dans les salles de classe. Également, l'utilisation d'exemples de la "vraie vie" ou du domaine d'étude des étudiants pourrait être pertinente afin d'accroître 
leur motivation à suivre le cours de statistique. Favoriser des questions “anonymes”, structurer le cours de façon à décourager la procrastination et effectuer des jeuxquestionnaires chaque semaine sont d'autres exemples de recommandations possibles. L'anxiété statistique est un phénomène prévalent, récurrent et "universel” auquel il est important de s'attarder.

\section{La période de questions}

1. Le fait de prendre plaisir à la tâche semble être un facteur important à la motivation de l'étudiant. Est-ce qu'il est possible que l'outil puisse intervenir dans la motivation à effectuer une tâche?

2. Il y a une réalité actuellement qui se traduit par une difficulté qu'éprouvent les étudiants à compter (l'innumérisme). Comment améliorer cette habileté à compter?

3. Quelle est la source de cette anxiété? Provient-elle de la perception des statistiques où ce sont les notions en soi qui provoquent cette source anxiogène?

L'anxiété statistique débuterait tôt dans l'éducation alors que les étudiants découvrent les premières notions mathématiques au primaire.

\section{Apprentissage des statistiques : limites cognitives}

Cette présentation de Denis Cousineau (Université d'Ottawa) propose une hypothèse de l'anxiété conséquente de sorte que l'anxiété liée aux statistiques émergerait au fil des ans parce que la compréhension des statistiques requiert un ou des processus déficients. Un lien possible serait que l'anxiété statistique s'exprimerait comme la dernière manifestation d'anxiétés plus anciennes telle que l'anxiété liée aux mathématiques (qu’on peut observer vers 10 ans). Il y aurait donc un processus déficient pour les étudiants souffrant d'anxiété statistique. La statistique étant la science du hasard, il est primordial de comprendre ce concept. Selon le présentateur, il faut des aptitudes de visualisation pour le comprendre afin de compter, de comparer un nombre à 5 et d'effectuer la rotation mentale. Il y a un lien systématique entre l'aptitude visuospatiale et l'anxiété mathématique et ce dernier est causal. Ce lien existe-t-il en ce qui a trait à l'anxiété statistique? Cela reste à confirmer. Il faudrait donc clarifier les liens entre l'anxiété statistique, l'anxiété mathématique et l'anxiété spatiale en démontrant les liens corrélationnel, causal et développemental de ces derniers. À ce sujet, il existe depuis peu un questionnaire d'anxiété spatiale, mais ce dernier n'est pas validé en français. De plus, il serait pertinent de pallier au phénomène de l'anxiété statistique avec des exercices de visualisation. La création d'un graphique parlant pour l'échantillonnage ainsi que pour l'estimation permettrait l'amélioration de la visualisation en statistique.
Également, il serait intéressant de connaître l'existence de concepts prérequis pour comprendre les statistiques.

\section{La période de questions}

1. La modélisation peut provoquer de l'anxiété chez les étudiants lorsqu'ils commencent à pratiquer cette technique, mais elle permet l'application des concepts théoriques.

2. Les étudiants sont habitués à traiter l'information de façon superficielle dans l'exécution de différentes tâches académiques. Par contre, il faudrait délaisser ce type de traitement de l'information et valoriser le traitement en profondeur. Pour ce faire, la création de modèles lorsqu'on lit des contenus statistiques pourrait en être un exemple. Plus ils sont actifs cognitivement, plus leur capacité d'imagination est sollicitée.

3. Quelques pistes de solutions peuvent être envisagées telles que la création de graphiques multidimensionnels ainsi que la pratique de méditation pleine conscience au premier cours de statistique de la session.

\section{L'enseignement des statistiques en sciences humaines et sociales : retour sur la matinée}

Cette section regroupe les propos discutés lors des échanges concernant les présentations de la matinée. À cet effet, il a été proposé que la nature du premier cours de statistique pourrait se traduire par une clarification des aspects positifs d'un cours de statistique au sein du parcours de l'étudiant. Concernant le choix des cours de type présentiel ou hybride, il a été convenu que la présence d'un professeur, donc l'apport de l'aspect humain au domaine des statistiques, est non-négligeable. Par contre, les tâches passives peuvent se dérouler à l'extérieur de la classe. Les séances de cours serviraient à des apprentissages actifs et à la présentation des contenus théoriques. Cela a engendré une discussion sur l'enseignement inversé qui consiste à favoriser le travail de l'étudiant et ne plus enseigner. Ce type d'enseignement comprend peu de cours théoriques, beaucoup de travaux d'équipe ainsi que des études de cas. Les données doivent provenir de ce qui les intéresse.

La motivation pour les étudiants suivant un cours de statistique est primordiale. Plusieurs techniques pourraient être mises de l'avant de sorte à accroître cette motivation. En effet, la création de capsules statistiques démontrant des réalités du domaine de la psychologie telle des nouvelles dans les médias ou des données présentées pour engendrer des raisonnements en sont de bons exemples. En d'autres mots, il faut se rapprocher de la pratique des gens à qui on enseigne. Les données doivent être réelles et authentiques. À titre d'exemple, l'action de faire mesurer des clous aux étudiants peut contribuer à 
l'enseignement de certaines notions statistiques telles que : aucune mesure n'est précise ni exacte, il existe des facteurs qui influencent l'erreur de mesure et il y a des règles imprécises. De plus, l'attitude du professeur a un impact considérable sur la motivation et l'expérience statistique des étudiants.

L'idée de l'utilisation d'un générateur de données aléatoires a été proposée dans les cours de statistique afin d'employer des contextes plus intéressants pour les étudiants. Cette discussion a mené les individus à aborder le logiciel statistique $\mathrm{R}$ qui est apprécié des enseignants et fantastique pour les études supérieures. Par contre, ce dernier comporte des problèmes importants dans le codage et la vérification de données.

Le dernier thème discuté était en lien avec les notions qui devraient être abordées dans le cours de statistique. Tous étaient unanimes au fait de présenter plus spécifiquement l'utilisation de bootstrap. De plus, l'enseignement de concepts mathématiques est un sujet mitigé chez les enseignants. À vrai dire, cela peut dépendre du style d'enseignement, mais selon eux, il faut qu'il y ait un minimum d'enseignement des mathématiques afin de comprendre les concepts sous-jacents aux statistiques. Cela a permis d'aborder les différentes méthodes d'évaluation en priorisant la diversité de celles-ci. En effet, la production d'un travail de session suivi d'un exposé oral où l'étudiant est amené à expliquer ses démarches ainsi que des examens visant à évaluer les connaissances semble être une formule adéquate. Dans le même ordre d'idée, la notion d'exercice demeure essentielle dans les cours de statistique visant à maximiser les interactions en classe ainsi que la compréhension par l'application de concepts généralement abstraits. Cela peut laisser place à un type d'approche par problème qui consiste à présenter une sortie sur le logiciel SPSS avec des données aberrantes ainsi que des données manquantes afin que les étudiants puissent effectuer des manipulations et développer leur autonomie. Cela leur permettrait de comprendre l'importance de la démarche. Finalement, les cours de statistique permettent de passer du rôle de candide ignorant à celui de futur professionnel capable de critiquer des articles, habileté essentielle à la poursuite des études universitaires.

\section{L'enseignement des statistiques en sciences humaines : les concepts essentiels}

Cette présentation de William Laporte (Université du Québec à Trois-Rivières) avait pour but de présenter les concepts jugés essentiels pour la compréhension des statistiques et à leur enseignement en sciences humaines selon des professeurs enseignant la statistique. Il y a plusieurs aspects qui ont été discutés dans la présentation. Par exemple, le rapport GAISE (2005) de l'American Sta- tistical Association (ASA) émet des recommandations sur ce que serait la littératie statistique, mais peu d'études empiriques s'intéressaient à la perception de professeurs concernant la pertinence des concepts essentiels et à leur intégration par les étudiants. Le projet de nature collaborative avait pour but de documenter les concepts clés que les professeurs en sciences humaines jugent essentiels à l'enseignement des statistiques. Le concept le moins pertinent pour les étudiants selon l'étude est la méta-analyse. L'étude s'adressait essentiellement aux professeurs qui ont enseigné des cours incluant de la statistique en sciences humaines.

Le projet était divisé en 2 phases. La première phase visait à recueillir, auprès des professeurs, les concepts jugés essentiels pour la littératie statistique en sciences humaines. La saturation empirique a été atteinte après seulement 34 participants. Les 354 réponses brutes obtenues ont ensuite nécessité un traitement de la part des chercheurs pour en arriver à une liste exhaustive, sans redondance et corrigée de 70 concepts. La phase 2 portait sur le classement des concepts essentiels selon leur priorité perçue et leur difficulté d'intégration par les étudiants (selon la perception des professeurs). Le questionnaire comportait deux échelles : l'une sur le degré de pertinence perçue du concept dans un premier cours de statistique à l'université dans la discipline du répondant et l'autre sur le pourcentage estimé d'étudiants qui suivent un premier cours universitaire de statistique dans la discipline du répondant qui parviennent à intégrer correctement le concept.

La nature exploratoire de l'étude ne permettait pas d'inférer les résultats à l'ensemble des professeurs enseignant la statistique. Néanmoins, les retombées de cette étude permettent de poser un regard critique sur la pratique et chaque professeur peut juger à son tour de ses méthodes et poser des questions au sein de la communauté de l'enseignement des statistiques. Également, les concepts les mieux intégrés sont les mesures de tendances centrales apprises plus tôt dans le parcours académique des étudiants. Il en ressort que les concepts qui semblent les plus pertinents en moyenne sont aussi ceux qui sont le mieux intégrés. L'inverse est aussi vrai. Les concepts jugés les moins pertinents en moyenne sont aussi ceux étant moins bien intégrés.

Il est d'autant plus important d'appliquer un contexte $\mathrm{au}$ problème ainsi qu'aux statistiques afin que l'étudiant puisse comprendre la pertinence des manipulations et ainsi diminuer son anxiété statistique. L’aspect du regard critique est considéré comme un acquis important du point de vue des enseignants qui se traduit par une consommation intelligente et citoyenne. Par contre, cet aspect semble être moins intégré par les étudiants. 


\section{La période de questions}

1. Il y a plus d'hommes que de femmes qui enseignent les statistiques de façon générale.

2. Il est difficile de déterminer l'état des connaissances des étudiants de la classe, car certains en sont à leur premier cours de statistique universitaire et d'autres non.

3. De quelle façon pourrait-on enseigner le développement d'un regard critique face aux statistiques?

Il serait intéressant de présenter des articles qui contiennent certaines lacunes en lien avec le contenu $\mathrm{du}$ cours. Par contre, ceux-ci sont difficiles à trouver, car ils sont généralement trop complexes ou rédigés en anglais. Une présentation orale pourrait être une solution et celle-ci pourrait permettre de transmettre des observations quant à des représentations graphiques ou des résultats anormaux.

4. Serait-ce une bonne idée de présenter la version d'un article bien conçu et la même comportant certaines lacunes?

Cela constitue des actions plutôt délicates, car il y a le nom de l'auteur sur l'article. Par contre, il y a des statistiques farfelues dans les journaux qui pourraient être présentées.

5. La technique du pharmachien afin de sensibiliser les gens à leur consommation de médicaments ainsi qu'à de fausses croyances concernant le domaine pharmaceutique pourrait-elle se transposer pour le domaine des statistiques?

6. Est-ce que l'éducation au cégep devrait débuter en sensibilisant les étudiants au sens critique?

\section{Propositions finales de ce colloque}

La formation d'un collectif sur l'enseignement des statistiques est une proposition qui comprendrait une rencontre annuelle qui aurait lieu au congrès de l'ACFAS ainsi qu'un lieu de dépôt (un wiki) pour les documents et les commentaires et suggestions concernant les méthodes d'enseignement. Ce collectif pourrait ultimement faire des demandes de subventions et publier des vignettes éducatives dans la revue TQMP. De plus, la création d'une plateforme visuelle adressée aux enseignants de statistique à travers le monde pourrait constituer une autre proposition intéressante. Dans le même ordre d'idée, un "Subject pool” pourrait être créé afin de favoriser le domaine de la recherche en statistique. À titre d'exemple, les étudiants qui seraient intéressés à participer aux études pourraient se voir attribuer un point bonus dans leurs cours de statistique.

Sébastien Béland se propose d'organiser le prochain colloque en 2019. Il demande s'il y a des suggestions de thèmes quant au colloque de l'an prochain. On propose (1) L'existence d'une formule gagnante pour l'enseignement des statistiques; (2) Les logiciels statistiques (SPSS, R et SAS); (3) Les méthodes d'évaluations dans les cours de statistique; et (4) Des statisticiens qui enseignent les statistiques.

\section{Citation}

LEMAY, C. \& LAPORTE, W. (2018). Compte rendu du colloque "L'enseignement des statistiques en sciences humaines et sociales”. The Quantitative Methods for Psychology, 14(3), 159-166. doi :10.20982/tqmp.14.3.p159

Copyright ( 2018 , LEMAY et LAPORTE. This is an open-access article distributed under the terms of the Creative Commons Attribution License (CC BY). The use, distribution or reproduction in other forums is permitted, provided the original author(s) or licensor are credited and that the original publication in this journal is cited, in accordance with accepted academic practice. No use, distribution or reproduction is permitted which does not comply with these terms.

Received: 10/06/2018 Accepted: 15/06/2018 